\title{
The methodology of teaching French language as a foreign in Russia and French-Russian education in cyberspace. Challenges and difficulties needed to skip in secondary school and higher education institute
}

\section{[La didactique du FLE en Russie et l'espace éducatif virtuel franco- russe. Perspectives et difficultés à surmonter à l'école secondaire et supérieure]}

\author{
Svetlana Kashchuk
}

DOI: 10.18355/XL.2018.11.01XL.27

\begin{abstract}
The article introduces the results of researches on the question of effective integration of Information and Communication Technologies into French language teaching for use in Russian secondary school and higher education institute.

Today society of the 21 st century needs skilled people in using foreign languages easily in real life as well as in cyberspace. Consequently, to create the third millennium people we need professors armed with linguistic multimedia competence that allows them to apply ICT into foreign language teaching in effective manner. The analysis shows that the professors of French in Russia lacked with pedagogic approaches allowing the usage ICT at French lessons.

Practice shows that the most effective pedagogic approach is a combination of new ICT and the methods that proved its effectiveness for centuries, for instance, problem solving learning or project-based learning.

In this article we introduce our experience which leads to successful education by means of organized cyberspace learning project between Russian and French students. Key words: cyber school, ICT in the education of FLT (French language teaching as foreign), didactics of FLT, project-based learning

Résumé

L'article présente ici les résultats des recherches consacrées à la question d'intégration efficace des technologies de l'information et de la communication (TIC) dans l'enseignement du français à l'école secondaire et supérieure russe.

La société du 21 ème siècle a besoin aujourd'hui des gens sachant utiliser facilement les langues étrangère dans la vie réelle aussi bien que sur l'espace virtuel. Donc, pour former ces citoyens du troisième millénaire, on a besoin des professeurs dotés d'une compétence professionnelle langagière multimédia qui leur permettra d'introduire les TIC dans l'enseignement des langues d'une façon efficace.

L'analyse faite auprès des professeurs de français en Russie à bien montré le manque d'approches pédagogiques permettant l'intégration des TIC aux cours de français. Comme l'a bien montré la pratique, le plus efficace c'est l'approche pédagogique réunissant les atouts des nouvelles TIC avec les méthodes qui ont bien montré leur efficacité depuis des siècles comme, par exemple, apprentissage par résolution des problèmes, l'apprentissage en collectivité ou apprentissage par projet.

Dans cet article nous présentons notre expérience qui nous a permis de mener à succès l'apprentissage par projet organisé sur l'espace éducatif virtuel franco-russe. Mots-clés: cyber école, TIC dans l'enseignement du FLE, français langue étrangère, didactique du FLE, apprentissage par projet word
\end{abstract}

\section{Introduction}

La problématique du présent article concerne la recherche sur les savoir-faire des professeurs de FLE d'intégrer les TIC dans le processus éducatif en Russie. On 
présente la définition de la compétence professionnelle langagière multimédia des professeurs, la graille de son évaluation aussi que les approches en didactique des langues permettant réinventer des pratiques pédagogiques à l'aide d'efficace association des TIC, notamment des applications Web 2.0 (blogs), avec les méthodes classiques. C'est un sujet important parce qu'on ne peut plus ignorer aujourd'hui le rôle que jouent les TIC dans tous les domaines de la vie contemporaine en général et dans l'enseignement des langues en particulier.

La didactique des langues c'est la science assez stricte qui définit avant tout les buts d'apprentissage, puis les approches pédagogiques permettant d'atteindre ces buts et enfin, les outils d'apprentissage (par exemple, les manuels, les technologies de l'information et de la communication dans l'enseignement (TICE), les services Web 2.0 etc.). Si le professeur fait le bon choix de toutes ces composantes du processus éducatif, l'apprenant à toutes les chances de réussir et d'atteindre le niveau désiré en langue étrangère. Et c'est surtout l'approche ou les approches sur lesquels est basée la construction des cours qui jouent le rôle clé dans l'enseignement des langues. Aujourd'hui, on parle beaucoup d'approche communicative (Berard, 1991), d'approche actionnelle (Griggs, 2010), approche interactionnelle (Ollivier, 2010) ou approche par les tâches (Rosen, 2009). Mais il ne faut pas non plus oublier l'approche éclectique, quand on prend ce qui marche bien dans tel ou tel méthode ou époque, ce que C. Puren a appelé «la didactique des langues étrangères à la croisée des méthodes » (Puren, 1994). Donc, la didactique des langues c'est simple et difficile en même temps, il faut faire un bon choix.

Commençons par les buts. Pourquoi apprend-on aujourd'hui les langues étrangères? Forcément, pas pour le plaisir de la lecture ou de la visualisation des films en langue des œuvres littéraires ou cinématographiques. D'après «Cadre européen commun de référence pour les langues" les buts d'apprentissage des langues étrangères aujourd'hui sont: l'acquisition des compétences linguistiques et des compétences générales (CECRL, 2001). Mais la cible essentielle, c'est la possibilité d'apprendre à étudier, à travailler, à vivre et à communiquer facilement dans le pays étranger (Bourgiognon, 2010, Richer 2010) nous ajoutons sur son territoire réel aussi bien que sur son espace langagier numérique. Et là, il nous manque une compétence - c'est une compétence langagière multimédia. Cette compétence permet aux apprenants des langues étrangères d'utiliser à son gré l'espace numérique du pays c'est-à-dire organiser la recherche, l'analyser et faire la synthèse des informations fiables aussi que de recevoir, stocker et transmettre les informations en langue étrangère pour résoudre les tâches communicatives.

Pour former les apprenants des langues étrangères capables d'utiliser pleinement dans leurs études et futures activités professionnelles l'espace langagier réel et numérique du pays étranger, il faut avoir les professeurs des langues étrangères à la hauteur de cette demande. Il faut que ces professeurs sachent intégrer efficacement les TIC dans le processus d'enseignement. Il faut qu'ils aient une compétence professionnelle multimédia langagière (Kashchuk, 2013). Cette compétence comprend deux composantes:

- les savoir-faire de professeur qui lui permettront d'utiliser dans la vie réelle et professionnelle les TIC de pointe en tant que les outils de communication ;

- les savoir-faire en didactique des langues, permettant de construire la leçon de langue étrangère en utilisant tous les atouts possibles des technologies du XXIème siècle.

Comme le souligne bien les spécialistes en didactique des langues, jusqu'aujourd'hui, nous avons beaucoup entendu parler de la nécessité d'intégrer les TIC dans l'enseignement des langues (Nazarenko, 2008 ; Ollivier, 2011). On réfléchit beaucoup sur les compétences des professeurs capables d'intégrer efficacement les TIC dans l'enseignement des langues (Karsanti, Kozarenko, 2016). Le problème du choix des 
sources et des applications Web 2.0 reste très actuel (Mangenot, 2010 ; Sysoev 2008). Il y a beaucoup d'expériences positives dans ce domaine, mais beaucoup plus de choses restent à réfléchir et on parle même de la réinvention des pratiques pédagogique d'intégration des TIC dans l'enseignement des langues (Guichon, 2012). Louis Porcher, grand didacticien français, a dit que «l'évaluation est la clef de l'enseignement»(Porcher, 2001). Il est aussi important d'élaborer la grille d'autoévaluation pour rendre les apprenants plus autonome dans leurs études (Veltcheff, 2003).

Donc, la première étape de nos recherches consistait à définir le problème, c'est-à-dire d'évaluer les composantes de la compétence professionnelle langagière multimédia des professeurs pour voir ce qui n'allait pas. Nous avons travaillé avec une soixantaine de professeurs de français de la région de Moscou qui ont suivi les cours de la formation continue "Les Technologies de l'information et de la communication dans l'enseignement du français à l'école russe»' de 2012 à 2016 par groupe de 15 personnes.

La grille d'évaluation des niveaux de lange étrangère CECRL aussi que la grille d'évaluation du «Brevet informatique et internet (B2i) ${ }^{1} »$ nous ont donné une idée de faire notre grille d'évaluation de la compétence professionnelle langagière multimédia des professeurs qui nous a permis d'évaluer :

1. les savoir-faire des professeurs qui leur permettent d'utiliser dans la vie réelle et professionnelle les TIC de pointe (niveau A dans la grille) ;

2. les savoir-faire en didactique contemporaine, les approches pédagogiques dans l'intégration des TIC aux cours de français (niveau B dans la grille).

\section{Utilisation des TIC en tant qu'outil de communication}

Niveau A

\begin{tabular}{|c|c|c|}
\hline Niveau & Description & $\begin{array}{l}\text { Les savoir-faire } \\
\text { prérequis }\end{array}$ \\
\hline $\begin{array}{l}\text { A1 } \\
\text { Maîtrise des fonctions de } \\
\text { bases, des procédés et des } \\
\text { règles qui permettent de } \\
\text { traiter l'information à l'aide } \\
\text { d'ordinateur et du réseau } \\
\text { Internet, aussi que la } \\
\text { possibilité de résoudre des } \\
\text { simples problèmes qui } \\
\text { apparaissent au cours de } \\
\text { travail sur l'ordinateur. }\end{array}$ & $\begin{array}{l}\text { Les savoir-faire tels que : } \\
\text { - allumer / éteindre l'ordinateur ; } \\
\text { - utiliser les logiciels ; } \\
\text { - recevoir, sauvegarder, } \\
\text { transmettre les informations ; } \\
\text { - imprimer les documents ; } \\
\text { - gérer et organiser des fichiers ; } \\
\text { etc... }\end{array}$ & - \\
\hline $\begin{array}{l}\text { A2 } \\
\text { Maîtrise du métalangage } \\
\text { des TIC et multimédia, les } \\
\text { savoir-faire dans le } \\
\text { domaine «environnement } \\
\text { informatique ». }\end{array}$ & $\begin{array}{l}\text { Exemple du métalangage des TIC: } \\
\text { le fichier, le dossier, le serveur, la } \\
\text { carte-mère, mémoire RAM, les } \\
\text { périphériques, l'imprimante, les } \\
\text { applications Web } 2.0 \text {, les liens } \\
\text { hypertextes, l'espace numérique, } \\
\text { les réseaux, le courriel etc. }\end{array}$ & Al \\
\hline
\end{tabular}

${ }^{1}$ Le brevet informatique et internet (B2i), 2011. http://eduscol.education.fr/cid46073/b2i.html 


\begin{tabular}{|c|c|c|}
\hline & $\begin{array}{l}\text { Organiser son environnement } \\
\text { informatique. Envoyer et recevoir } \\
\text { les courriels. }\end{array}$ & \\
\hline $\begin{array}{l}\text { A3 } \\
\text { Les savoir-faire dans } \\
\text { le domaine } \\
\text { bureautique. }\end{array}$ & $\begin{array}{lr}\text { Créer, produire, traiter, exploiter } \\
\text { des données des documents } \\
\text { numériques contenants les } \\
\text { tableaux et les graphiques. Savoir } \\
\text { scanner et sauvegarder les } \\
\text { documents. L'illustration des } \\
\text { documents avec Powerpoint. }\end{array}$ & $\mathrm{A} 1, \mathrm{~A} 2$ \\
\hline $\begin{array}{l}\text { A4 } \\
\text { Maîtrise de l'espace } \\
\text { numérique, des } \\
\text { réseaux sociaux et } \\
\text { professionnels. }\end{array}$ & $\begin{array}{l}\text { Se connecter aux différents types } \\
\text { de réseaux. Pouvoir s'identifier sur } \\
\text { les réseaux sociaux et } \\
\text { professionnels. } \\
\text { Pouvoir trouver les sources } \\
\text { d'informations fiables. Se sentir à } \\
\text { l'aise dans le monde numérique en } \\
\text { langue maternelle et étrangère. }\end{array}$ & $\mathrm{A} 1, \mathrm{~A} 2, \mathrm{~A} 3$ \\
\hline $\begin{array}{l}\text { A5 } \\
\text { Utilisation } \\
\text { multimédias, des } \\
\text { applications Web } 2.0\end{array}$ & $\begin{array}{l}\text { Créer les documents multimédias } \\
\text { en utilisant les appareils photos et } \\
\text { les caméras numériques. } \\
\text { Utilisation libre des applications } \\
\text { Web } 2.0 \text { pour gérer, sauvegarder } \\
\text { et transmettre les informations } \\
\text { sous le format numérique } \\
\text { multimédia. }\end{array}$ & $\begin{array}{l}\text { A1, A2, } \\
\text { A3, A4 }\end{array}$ \\
\hline
\end{tabular}

\section{Utilisation des TIC en tant qu'outil d'enseignement}

Niveau B

\begin{tabular}{|l|l|l|}
\hline Niveau & Description & $\begin{array}{l}\text { Les } \\
\text { savoir- } \\
\text { faire } \\
\text { prérequis }\end{array}$ \\
\hline B1 & $\begin{array}{l}\text { Organisation et personnalisation de } \\
\text { l'espace éducatif numérique des } \\
\text { apprenants. }\end{array}$ & $\begin{array}{l}\text { A1, A2, } \\
\text { Le professeur doit être capable de } \\
\text { gérer les situations difficiles, les } \\
\text { problèmes techniques qui vont } \\
\text { en didactique } \\
\text { permettant au } \\
\text { professeur de } \\
\begin{array}{l}\text { comprendre son } \\
\text { rôle en classe } \\
\text { informatique de } \\
\text { langue. }\end{array} \\
\text { langue. Le professeur doit adopter } \\
\text { une attitude responsable en tant que } \\
\text { facilitateur d'apprentissage en classe } \\
\text { informatique de langues. }\end{array}$ \\
\hline
\end{tabular}

XLinguae, Volume 11 Issue 1XL, January 2018, ISSN 1337-8384, eISSN 2453-711X 


\begin{tabular}{|c|c|c|}
\hline $\begin{array}{l}\text { B2 } \\
\text { Les savoir-faire en } \\
\text { didactique permettant aux } \\
\text { apprenants de s'approprier } \\
\text { un environnement } \\
\text { informatique en classe de } \\
\text { langue. }\end{array}$ & $\begin{array}{l}\text { Le professeur enseigne aux élèves } \\
\text { comment trouver, analyser, } \\
\text { synthétiser, traiter, exploiter des } \\
\text { données numérique, aussi que } \\
\text { comment créer ses propres documents } \\
\text { en langue étrangère en utilisant les } \\
\text { différents formats, liens hypertextuels, } \\
\text { images etc. } \\
\text { Enseigner le respect du Netiquette, } \\
\text { des droits d'auteurs sur la Toile. }\end{array}$ & $\begin{array}{l}\text { A1, A2, } \\
\text { A3, A4 }\end{array}$ \\
\hline $\begin{array}{l}\text { B3 } \\
\text { Les savoir-faire } \\
\text { en didactique } \\
\text { permettant de } \\
\text { trouver, analyser, } \\
\text { intégrer dans le } \\
\text { processus } \\
\text { éducatif les } \\
\text { ressources } \\
\text { didactiques } \\
\text { d'Internet. }\end{array}$ & $\begin{array}{l}\text { Le professeur doit être } \\
\text { d'analyser les logable } \\
\text { programmes autonomes du point de } \\
\text { vue de leur efficacité pour le processus } \\
\text { éducatif: } \\
\text { - quelles sont les approches } \\
\text { didactiques de base de ces programmes } \\
\text { et ces logiciels; } \\
\text { - quels dont les principes de leur } \\
\text { intégration aux cours de langue ; } \\
\text { - sont-ils difficile/facile à utiliser par } \\
\text { les apprenant; } \\
\text { - sont-ils compatibles avec les buts } \\
\text { d'apprentissage de langue; } \\
\text { - font-ils gagner ou perdre du temps } \\
\text { aux cours de langue etc.... }\end{array}$ & $\begin{array}{l}\mathrm{A} 1, \mathrm{~A} 2, \\
\mathrm{~A} 5, \\
\mathrm{~B} 1, \mathrm{~B} 2\end{array}$ \\
\hline $\begin{array}{l}\text { B4 } \\
\text { Les savoir-faire } \\
\text { en didactique } \\
\text { permettant } \\
\text { d'intégrer le } \\
\text { multimédia dans } \\
\text { les différents } \\
\text { aspects } \\
\text { d'apprentissages } \\
\text { de langue } \\
\text { (compréhension } \\
\text { écrite, } \\
\text { compréhension } \\
\text { orale, production } \\
\text { écrite, production } \\
\text { orale } \\
\text { (monologue, } \\
\text { dialogue). }\end{array}$ & $\begin{array}{l}\text { Le professeur doit savoir organiser le } \\
\text { travail individuel et en groupes en } \\
\text { classe informatique de langue avec } \\
\text { l'intégration efficace des TIC pour } \\
\text { former les compétences } \\
\text { communicatives langagières des } \\
\text { apprenants. }\end{array}$ & $\begin{array}{l}\text { A } 1-5 \text {, } \\
\text { В } 1-3\end{array}$ \\
\hline $\begin{array}{l}\text { B5 } \\
\text { Connaître les } \\
\text { approches } \\
\text { didactiques } \\
\text { permettant }\end{array}$ & $\begin{array}{l}\text { Le professeur doit savoir construire les } \\
\text { cours de langue dans l'espace réel et } \\
\text { numérique bien en accord avec le but } \\
\text { d'apprentissage. Le professeur doit } \\
\text { enseigner aux apprenants les }\end{array}$ & $\begin{array}{l}\text { A } 1-5 \text {, } \\
\text { В } 1-4\end{array}$ \\
\hline
\end{tabular}




\begin{tabular}{|l|l|}
\hline l'intégration des & $\begin{array}{l}\text { possibilités de communiquer en langue } \\
\text { étrangère via les applications Web 2.0 } \\
\text { atouts des TIC et } \\
\text { multimédia aux } \\
\text { cours de langue. }\end{array}$ \\
didactique classique (apprentissage par \\
projet, apprentissage par résolution des \\
problèmes, apprentissage en commun) \\
avec les possibilités des technologies \\
de communication numériques de \\
pointe. Cela permettra d'enseigner la \\
langue étrangère presque dans les \\
conditions authentiques dans lesquelles \\
cette langue sera ensuite utilisée par \\
les apprenants pour la communication \\
professionnelle ou privée.
\end{tabular} \mid

Votre niveau de la compétence professionnelle langagière multimédia

\begin{tabular}{|l|l|l|l|l|l|}
\hline & A1 & A2 & A3 & A4 & A5 \\
\hline $\begin{array}{l}\text { Utilisation des TIC en tant } \\
\text { qu'outil de communication }\end{array}$ & & & & & \\
\hline & B1 & B2 & B3 & B4 & B5 \\
\hline $\begin{array}{l}\text { Utilisation des TIC en tant } \\
\text { qu'outil d'enseignement }\end{array}$ & & & & & \\
\hline
\end{tabular}

Les sondages faits auprès des professeurs de français de la région de Moscou ont bien montré que le niveau A, l'utilisation des TIC en tant que le moyen de communication est assez élevé : $53 \%$ des professeurs ont eu le niveau le plus élevé A5, $17 \%$ - le niveau A4, $6 \%$ - A3, $6 \%$ - A2, $6 \%$ - A1. $12 \%$ n'ont pas d'ordinateur.
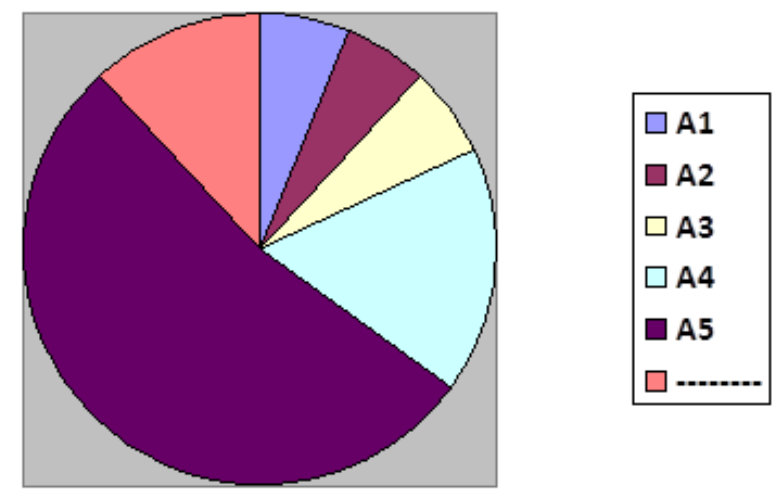

Image 1. Diagramme représentant le niveau d'utilisation des TIC en tant qu'outil de communication par les professeurs de français dans les écoles secondaires en région de Moscou. 
Contrairement à cette situation, la deuxième composante de la compétence professionnelle multimédia langagière des professeurs est beaucoup moins développée : $41 \%$ des professeurs n'intègrent d'aucune façon les TIC dans ses cours de français, $30 \%$ ont eu le niveau très bas B1, $6 \%$ - B2, $6 \%$ - B3 et seulement $17 \%$ - B4. Parmi les sondés personne n'a eu le niveau B 5, ne niveau le plus élevé.
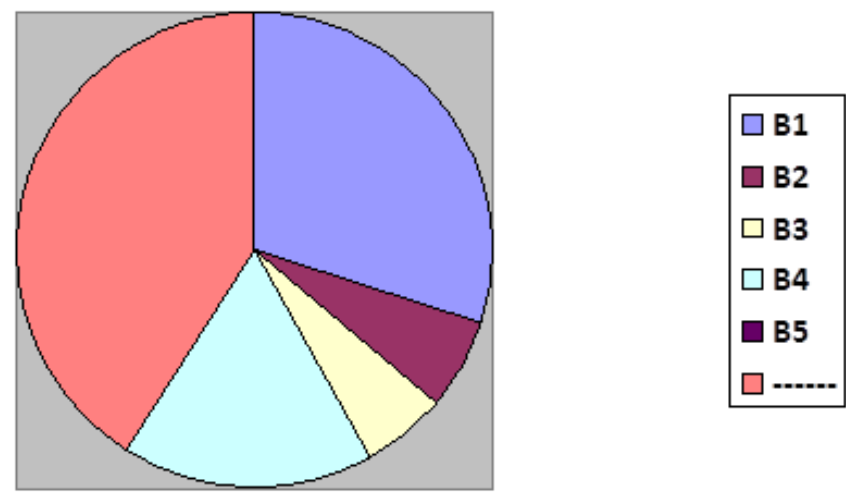

\section{Image 2. Diagramme représentant le niveau d'utilisation des TIC en tant qu'outil d'enseignement par les professeurs de français dans les écoles russes en région de Moscou.}

Quelle conclusion tirer de tout cela? Des spécialistes en didactique des langues (Kozarenko, 2015 ; Metzger, 2011) soulignent que la didactique des langues a besoin aujourd'hui de nouvelles idées, de nouvelles possibilités permettant aux professeurs de construire leurs cours de langues étrangères à la hauteur de la demande de la société contemporaine, la société d'information, la société qui se développe et communique en langues étrangères dans la vie réelle aussi bien que sur l'espace langagier numérique.

Dans le cadre de nos recherche, nous avons essayé de résoudre le problème de manque d'approche en pédagogie contemporaine d'intégration efficace des TIC dans l'enseignement des langues en associant les atouts des nouvelles TIC avec une des méthodes classiques, l'apprentissage par projet.

\section{L'expérience scolaire}

En exemple, nous vous présentons le projet scolaire « Cyberécole » qui a été réalisé la classe russe où le français était enseigné comme LV1.

Ce projet a été réalisé à succès à l'école secondaire russe de la petite ville de Kolomna (la région de Moscou). Il y avait un groupe de 20 élèves de 13-15 ans qui suivaient les cours scolaires de français LV1 pendant 3 ans (deux cours de 45 minutes par semaine). Les étapes du projet étaient suivantes :

$$
\text { 1. Le choix du nom du projet. }
$$

Il est important d'organiser la discussion entre les élèves à ce sujet. Dans notre cas, les enfants ont dressé toute une liste des noms possibles en utilisant pleinement le lexique du sujet «mon école », par exemple : «L'école virtuelle», "L'école sur la Toile », «La Web amitié », " La cyber amitié scolaire» etc. Nous avons choisi la 
«Cyberécole12» (12 - parce qu'en Russie tous les établissements scolaires ont leurs numéros). Un peu plus tard, cette expérience s'est étendue sur une autre école, ce qui a donné le projet « Cyberécole 17 ».

2 . Le choix de la plateforme du projet.

Aujourd'hui, les blogs sont largement présents dans les approches non ordinaires de l'enseignement des langues (Marchal, 2010 ; Pradal, 2007). Nous avons choisi le blogue comme le cyber endroit des rencontres virtuelles, parce que c'est le service du Web 2.0 qui est très facile à utiliser (on a juste besoin de quelques minutes pour créer son blogue), facile à gérer et bien sécurisé (si on n'a pas un code d'accès, on ne peut rien écrire sur votre blogue). L'utilisation efficace des blogs dans le processus éducatif est prouvé par plusieurs études (Louveau, 2006, Ollivier, 2010). Ce qui m'a plu personnellement dans le travail avec le blogue, c'est que j'ai pu gérer les brouillons de mes élèves de n'importe quel endroit. Même quand j'ai dû partir pour quelques semaines en mission à une autre ville, j'avais toujours la possibilité de continuer notre projet.

3. La présentation du groupe d'élèves, des professeurs et de nos intentions.

Avant de passer à l'étape de la recherche des partenaires sur le Web, il fallait faire une présentation pour montrer qui nous sommes: nous avons mis sur notre blogue quelques photos de notre groupe (en classe et devant l'école) aussi que quelques photos de notre petite ville de Kolomna (ancienne et très jolie).

4. La recherche des partenaires.

Cette étape est assez difficile et imprévisible car il faut compter sur la chance. Trouver les partenaires du même âge que mes élèves et aussi intéressés par notre projet n'était pas si simple. Cependant, ce problème a été résolu assez rapidement, car il y a un grand cyber espace didactique sur la Toile francophone. J'ai posté mon annonce sur le site de «Franc-parler» (http://www.francparler-oif.org/) la rubriques «Correspondance de classe» ${ }^{2}$ et $\mathrm{j}$ 'ai eu très vite la réponse de mon collègue français, professeur du lycée intéressé par notre projet. Il est à souligner que les buts des professeurs dans ce projet étaient tout à fait différents: le professeur français voulais apprendre à ses élèves la multiplicité du monde en leur montrant le pays assez mystérieux comme l'est la Russie pour les Français, tandis que moi, le professeur russe voulait rendre les cours de FLE dans ma classe plus efficaces et attractifs, et par conséquent, motiver les élèves à apprendre le français. Mais cela ne nous a pas empêché de travailler bien ensemble, parce que ce que nous avions en commun, c'était le désir de partager nos cultures et organiser un vrai échange humain entre les élèves.

5. L'élaboration de la stratégie.

Cette étape consiste à élaborer la fréquence de nos publications sur le blogue et le contenu qui d'un côté sera intéressant aux élèves des deux pays, et d'autre part, restera dans le cadre des cours de français du programme scolaire obligatoire russe. La fréquence se limitait à un cours par semaine pendant lequel on publiait nos textes et nos photos. Mais il faut y ajouter le travail préparatif hors la classe. Les élèves travaillaient toute une semaine sur leur matériel: ils publiaient les textes dans leurs « brouillons » virtuels du blogue ou je les corrigeais et renvoyais à l'élève si le travail était mal fait. Après le travail sur les fautes, si le texte était bien fait, je le publiais sur le blog, se qui signifiait pour l'élève une bonne note.

6. Le déroulement du projet.

${ }^{2}$ Malheureusement, cette rubrique n'existe plus, mais le site reste fiable et très intéressant du point de vue des matériaux didactique pour les professeurs de FLE. XLinguae, Volume 11 Issue 1XL, January 2018, ISSN 1337-8384, eISSN 2453-711X 
Une fois par semaine ma classe travaillait dans la salle informatique où nous lisions les textes de nos correspondants français et publions les nôtres en les illustrant avec les photos. A la fin de la leçon, les enfants recevaient le devoir pour la prochaine fois. Je donnais les devoirs en fonction de ce que présentait la dernière communication sur le blogue : si les élèves français s'intéressaient à quelque chose de précis (par exemple quelle musique écoutent les correspondants russes? ou auxquels jeux vidéo est-ce qu'ils jouent? etc.), les élèves russes devaient préparer les réponses, sinon, on discutait les sujets de notre programme scolaire choisi d'avance.

Il est à noter, qu'en réalisant le projet, nous avons rencontré une difficulté de la « sympathie » entre les élèves qui voulaient communiquer plus avec telle ou telle personne. Notre projet pédagogique se transformait petit à petit du grand projet entre deux classes en quelques plus petits projets entre deux personnes se sympathisant. Cette situation mettait le désordre dans le contenu du blogue où les élèves de deux nationalités s'envoyaient les petits messages. D'une part c'était notre réussite, nous avons pu organiser une vraie communication, mais d'autre part, il fallait continuer le projet officiel. La solution a été trouvée : nous avons demandé aux élèves de créer leurs propres blogues et continuer la correspondance privée sur leur cyberespace personnel.

7. L'évaluation du projet.

Ce stade est assez important. Comment comprendre si le projet de la communication virtuelle est bien réussi ? Nous avons pensé au CECRL où on distingue bien les compétences générales et les compétences langagières. Donc, nos élèves devaient apprendre à :

expérience de la vie ;

communiquer en utilisant leurs savoirs générales, savoir-être et

utiliser le français comme instrument de communication en montrant la compétence linguistique, sociolinguistique et pragmatique.

On peut dire que notre projet a bien réussie car, premièrement, la communication a bien passé entre les deux classes et, deuxièmement, elle a bien passé en français, langue de communication.

A la fin de l'année scolaire, nous avons demandé à nos élèves de décrire en quelques phrases ce qu'ils pensent du projet « Cyberécole ». Voilà quelques impressions :

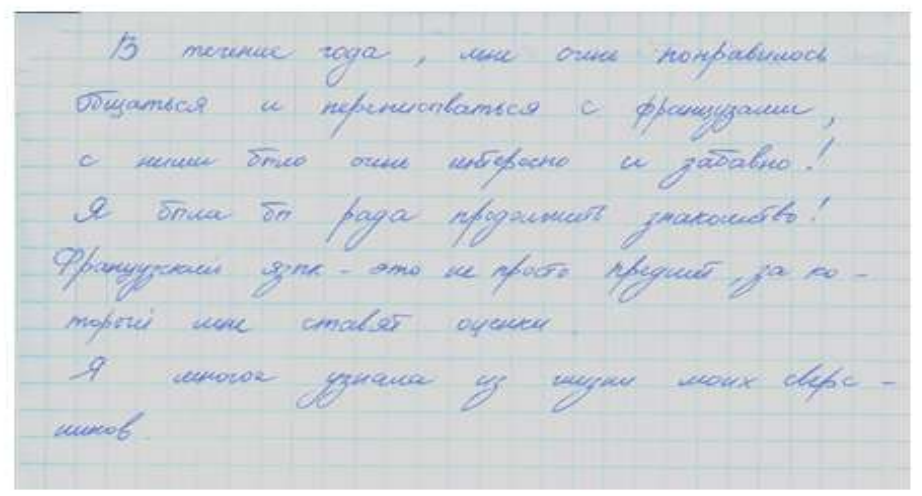

\section{Image 3. Impression du projet d'un des élèves russes.}

( «J'ai beaucoup aimé de communiquer avec les Français pendant l'année scolaire. C'était intéressant et drôle ! J'aimerais continuer cette communication! Le français ce 
n'est pas seulement la matière scolaire pour laquelle je reçois les notes. J'ai surtout appris beaucoup de chose sur les enfants de mon âge. »

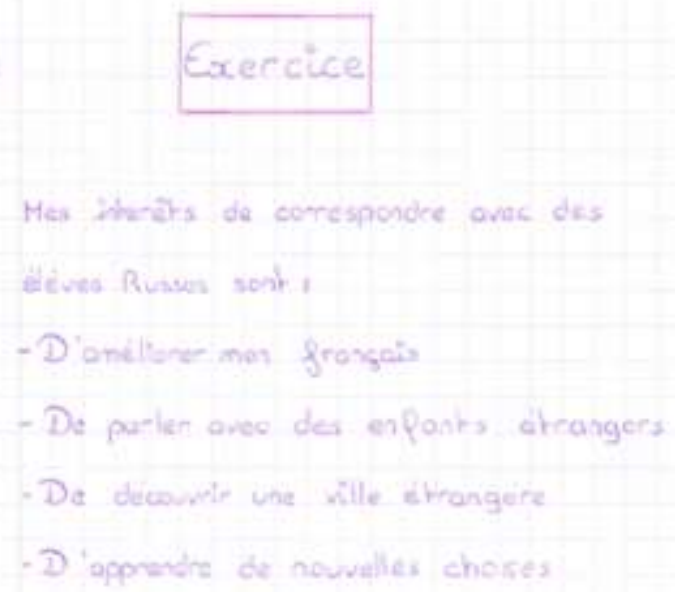

Image 4. Impression du projet d'un des élèves français.

(«Mes intérêts à correspondre avec des élève russes sont :

- d'améliorer mon français ;

- de parler avec des enfants étrangers ;

- de découvrir une ville étrangère ;

- d'apprendre de nouvelles choses. »)

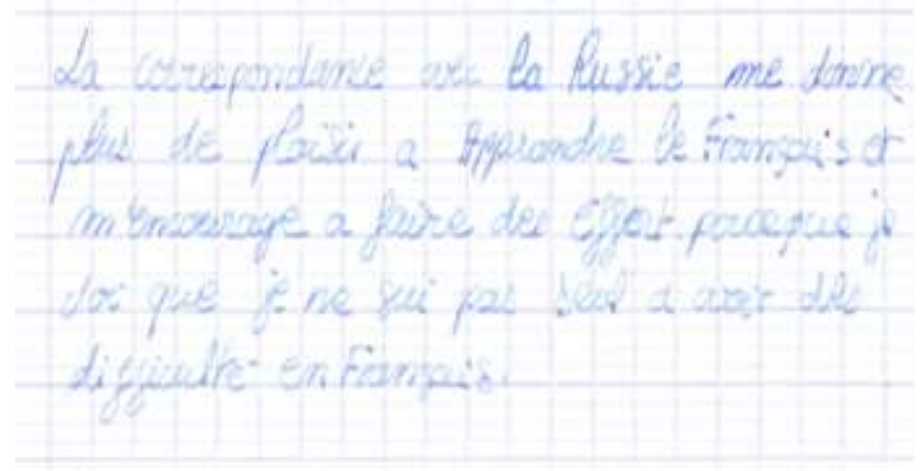

Image 5. Impression du projet d'un des élèves français.

( « La correspondance avec la Russie me donne plus de plaisir à apprendre le français et m'encourage à faire les efforts parce que je vois que je ne suis pas seul à avoir les difficultés en français !»)

Le projet « Cyberécole» (http://www.cyberecole12.blogspot.com) a duré 2 ans. Du point de vu pédagogique il peut être considéré comme un accompagnement didactique très efficace des cours de français à l'école. Pendant l'année scolaire, nous avions deux cours ordinaires et un cours dans la salle d'ordinateurs par semaine. Les sujets discutés avec les élèves français correspondaient bien avec ce qu'on faisait 
pendant les cours ordinaires. Nous avons choisi tels sujets comme «Présentation de ma ville natale», «Ma famille», «Problèmes avec les parents», «Fêtes et traditions en France et en Russie», «Ma vie scolaire» etc. Nous n'avons pas pu mettre sur la toile tous les sujets de conversation du programme scolaire russe (faute de temps). Il est à souligner que les sujets qui étaient travaillés de manière dite «classique» et puis discutés sur la Toile avec nos correspondants français étaient nettement mieux acquis par les élèves (dans les énoncés, il y avait beaucoup plus de liberté et le niveau de langue était plus élevé).

\section{L'expérience universitaire}

L'organisation de la communication virtuelle préoccupe aujourd'hui les professeurs des universités du monde, y compris en Russie (Hromenkov, 2014 ; Gusev, 2016 ; Popova N. 2017). Notre travail de recherche à l'Université suivait bien la direction de mise en pratique de l'intégration des applications Web 2.0, notamment, les blogs, au processus de formation des futures professeurs de français langue étrangère.

Le projet de la communication virtuelle francophone a été organisé à l'université pédagogique de Kolomna dans le cadre d'échange universitaire entre deux universités partenaires. Il y a une dizaine d'années qu'existe et fonctionne très bien un échange d'étudiants entre l'université de Caen (Normandie, France) et l'université de Kolomna (région de Moscou). Les étudiants partent pour quelques mois dans les universités partenaires en stage linguiste et pédagogique. Ces échanges apportent beaucoup sur le plan de l'étude et de l'apprentissage des modes de vie russe et française à toutes les deux universités mais, hélas, ils ne sont pas accessibles à tous les étudiants.

Etant à l'époque professeur de FLE et de la didactique des langues à l'université de Kolomna, j'ai travaillé avec les étudiants, futurs professeurs de français des écoles.

Nous avons eu l'idée d'offrir au plus grand nombre d'étudiants russes la possibilité de partir spirituellement en stage à Caen en créant un espace virtuel entre nos deux établissements. Aux cours de la didactique du FLE, nous avons créé un blogue «Stage en France» (http://francekgpi.blogspot.com) où les étudiants russes publiaient leurs impressions de la vie étudiante à Caen, ce qui a permis à ceux qui n'ont pas eu la chance de partir en France, de suivre presque en direct le stage, tout en restant en Russie.

Le projet «Stage en France» s'est très bien inscrit dans le cadre de la pédagogie active et l'approche par compétences du CECRL. Mais il a aussi eu les particularités russes dont la défense et la promotion de la langue française en tant que langue étrangère qui recule aujourd'hui devant l'anglais.

Le projet «Stage en France» a très bien fonctionné pendant deux années universitaires et a donné des résultats intéressants: le blogue des étudiant russes en France est devenu très populaire non pas seulement parmi les étudiants apprenant le français, mais ce qui est le plus étonnant, c'est que le choix de langue étrangère par les étudiants d'autres facultés (de lettres, d'économie, d'histoire etc.) a aussi été influencé vers le français.

$\mathrm{Au}$ fil du temps, notre espace francophone virtuel est devenu l'endroit de cyber rencontres de nos étudiants qui partaient en France ou en revenaient où ils partageaient leurs expériences, demandaient et donnaient des conseils. C'est un très bon exemple d'une des spécificités des technologies Web 2.0 - l'intelligence collective, à laquelle je peux ajouter la sentimentalité collective des étudiants et professeurs russes aimant la France, sa langue et sa culture.

Et pour conclure, en ce qui concerne la didactique du FLE en Russie, à part l'élaboration des nouvelles approches intégrant efficacement les TIC dans le processus d'enseignement à l'école secondaire et supérieure, il faut motiver les 
apprenants pour le choix du français en tant que langue étrangère. Malheureusement, la situation d' " anglobalisation ${ }^{3}$ " devient un facteur majeur qui influence de plus en plus le choix des étudiants et des écoliers.

Il est vrai que le français, comme d'autres langues européennes, devient de moins en moins présent sur le marché de travail russe. Actuellement, sur le marché russe il existe un manque de personnes qui parlent bien le français. Les spécialistes russes, sachant bien communiquer en français, sont recherchés et mieux payés que leurs collègues ne parlant que l'anglais.

Donc, la didactique du FLE en Russie vise aujourd'hui deux cibles essentielles: intégrer efficacement les TIC dans l'enseignement pour élaborer les cours dignes de l'université et de l'école du troisième millénaire et motiver les étudiants et les élèves à choisir le français en tant que langue étrangère comme matière scolaire et future langue étrangère professionnelle. Notre pratique a bien montré que ces deux buts peuvent être atteints par l'élaboration des approches nouvelles au cours du FLE. Dans notre cas, c'était l'organisation des projets pédagogiques de la cyber communication qui a permis aux écoliers russes d'utiliser le français comme outil de communication avec les écoliers français, tisser les relations humaines internationales, et aux étudiants russe en stage à Caen de partager leur expérience de la vie et des études en France avec ceux qui n'apprenaient le français que dans les salles d'ordinateurs à l'université.

\section{Bibliographic references}

BERARD, E. L'approche communicative. Théorie et pratiques. - Paris : CLE International, 1991. ISBN 2-19-033352-0.

BOURGUIGNON, C. 2010. Pour enseigner les langues avec CECPL. Paris: Delagrave Edition. ISBN : 978-2-206-01646-7.

Cadre européen commun de référence pour les langues: apprendre, enseigner, évaluer. 2001. Paris: Didier. Available online: https://www.coe.int/t/dg4/linguistic/Source/CECR_fr.pdf .

GUICHON N. 2012. Vers l'intégration des TIC dans l'enseignement des langues. Paris : Didier. ISBN : 978-2-278-07263-7.

GRIGGS, P. 2010. A propos de l'articulation entre l'agir de l'usage et l'agir de l'apprentissage dans une approche actionnelle : une perspective sociocognitive. Dans L'approche actionnelle dans l'enseignement des langues. Onze articles pour mieux comprendre et faire le point. Paris : Diffusion FLE. pp. 79- 101. ISBN 978-84-8443545-7.

GUSEV, D. FLEROV, O. 2016. K voprosu o mezhkul'turnoj kommunikacii v kontekste ehkonomiki obrazovaniya. Dans Nauka i shkola. № 2 ss. 30-39 ISSN 1819463X. Lire en ligne: http://nauka-i-shkola.ru/node/98 .

HROMENKOV, P. 2014. Issledovatel'skaya deyatel'nost' studentov pedvuza v usloviyah mezhdunarodnoj kommunikacii. Dans Prepodavatel' XXI vek. № 2, ss. 93 101. ISSN 2073-9613. Available online: https://cyberleninka.ru/article/n/issledovatelskaya-deyatelnost-studentov-pedvuza-vusloviyah-mezhnauchnoy-kommunikatsii .

KARSANTI, T. - KOZARENKO, O. M. Novaya model' informacionnyh kompetencij studentov i vozmozhnosti ee primeneniya $\mathrm{v}$ prepodavanii/izuchenii inostrannyh yazykov. In Lingvometodicheskie i psihologo-pedagogicheskie aspekty prepodavaniya professional'no orientirovannogo inostrannogo yazyka $\mathrm{v}$ vuze : monografiya koll. avtorov pod red. professora M.V. Mel'nichuk.-M.: Nauchnye

${ }^{3} \mathrm{~L}$ 'anglobalisation - terme inventé par l'auteur.

XLinguae, Volume 11 Issue 1XL, January 2018, ISSN 1337-8384, eISSN 2453-711X 
tekhnologii,.- M., 2016 .- S. 204-241, pages 204-241, Moskva. Moskva. ISBN 978-5-4443-0090-9.

KASHCHUK, S. 2013. Professional'naya mul'timedijnaya yazykovaya kompetenciya uchitelya inostrannogo yazyka. Dans Nauka i shkola, № 6, ss. 22-26, ISSN 1819463X. Available online: http://nauka-i-shkola.ru/2013-6.html .

KAZARENKO, O. 2015. Informacionnye tekhnologii v prepodavanii i izuchenii inostrannyh yazykov: informacionnaya kul'tura i veb-proektnaya deyatel'nost'. Dans Magiya INNO: novoe v issledovanii yazyka i metodike ego prepodavaniya Materialy Vtoroj nauchno-prakticheskoj konferencii. otv. red.: D.A. Kryachkov. Vol 2. Pp. 485489. Available online: http://elibrary.ru/item.asp?id=25247314 .

LOUVEAU, E. - MANGENOT, F. 2006. Internet et la classe de langues, Paris : CLE International. ISBN : 978-2-09-033069-4.

MANGENOT, F. 2010. Créer une banque de tâches Internet: quels descripteurs pour quelles utilisations ? Dans Etude de linguistique appliquée № 160 (numérique, et enseignement du français à l'université). pp. 433 - 444.

MARCHAL, B. 2010. Microblogue. Un outil d'interaction quasi simultanée entre apprenant et enseignant. Dans Le français dans le monde. № 368, pp. 28 - 29. ISSN 0015-9395.

METZGER, J.-L. 2011. Internet et pratiques professionnelles dans l'enseignement secondaire : quelles évolutions ? Dans Poyet F. \& Develotte C. (dir.) : L'éducation à l'heure du numérique : état des lieux, enjeux et perspectives, INRP, Lyon, coll. Technologies nouvelles et éducation, pp. 49-70.

OLLIVIER, C. 2010. Ecriture collaborative en ligne : une approche interactionnelle de la production écrite pour des apprenants acteurs sociaux et motivés. Dans Revue française de linguistique appliquée, vol. XV, n. 2, pp. 121 - 137. ISSN : 1386-1204.

PORCHER L. - BALLE, F. - MEIRIEU Ph. 2001. Expériences. Transferts. Construire une recherche. - Paris : CREDAM.

PRADAL, Fr. 2007. Avec le blogue, communiquez ! Le français dans le monde, № 351. pp. 30-31. ISSN 0015-9395.

PUREN, C. 1994. La didactique des langues étrangères à la croisée des méthodes. Essai sur l'éclectisme. - Paris : Didier,. - 203 p.

ROSEN, E. 2009. La perspective actionnelle et l'approche par les tâches en classe de langues. Dns Le français dans le monde, Recherches et Applications 45, Paris : Clé International pp. 6-14.

RICHER, J.-J. 2010. Lecture du Cadre : continuité ou rupture? L'approche actionnelle dans l'enseignement des langues. Douze articles pour mieux comprendre et faire le point. Paris : Diffusion FLE. pp. 13-49. ISBN 978-84-8443-545-7.

NAZARENKO, A. 2008. Lingvisticheskoe obrazovanie XXI veka: novye orientiry, novye formy. Dans Materialy mezhdunarodnoj nauchno-prakticheskoj konferencii «Informacionno-kommunikacionnye tekhnologii v obuchenii inostrannym yazykam i mezhkul'turnoj kommunikacii» Vypusk 3. - Moskva: MGU im. M. V. Lomonosova, s. $8-15$.

OLLIVIER, Ch. - PUREN, L. 2011. Le Web 2.0 en classe de langue. Paris: Edition maison des Langues. ISBN : 978-2-35685-077-5.

POPOVA, N. TRET'YAKOVA, G. 2017. Telekommunikacionnyj proekt kak realizaciya poliloga kul'tur $\mathrm{v}$ obuchenii inostrannomu yazyku $\mathrm{v}$ vuze. Dans Inostrannye yazyki v shkole, № 2, pp. 29-38. ISSN 0130-6073.

SYSOEV, P. - EVSTIGNEEV, M. 2008. Sovremennye uchebnye Internet-resursy v obuchenii inostrannomu yazyku. Inostrannye yazyki v shkole. № 6. pp. 1 - 10. ISSN 0130-6073.

VELTCHEFF, C. - HILTON, S. 2003. L'évaluation en FLE. Paris : HACHETTE Français langue étrangère. ISBN 2011552257. 
Words: 5302

Characters: 34905 (19,39 standard pages)

Prof. Dr .Svetlana Kashchuk

Foreign languages department

Administration faculty

Lomonossov Moscow State University

1-52, Leninskiye Gory, Moscow, GSP-1

119991

Russia

kashchuk@spa.msu.ru 